\title{
Encapsulation of Mentha Oil in Chitosan Polymer Matrix Alleviates Skin Irritation
}

\author{
Nidhi Mishra, ${ }^{1}$ Vineet Kumar Rai, ${ }^{1}$ Kuldeep Singh Yadav, ${ }^{1}$ Priyam Sinha, ${ }^{1}$ Archana Kanaujia, ${ }^{1}$ \\ Debabrata Chanda, ${ }^{2}$ Apurva Jakhmola, ${ }^{2}$ Dharmendra Saikia, ${ }^{2}$ and Narayan Prasad Yadav ${ }^{1,3}$
}

Received 13 June 2015; accepted 22 July 2015; published online 8 August 2015

\begin{abstract}
Mentha spicata L. var. viridis oil (MVO) is a potent antifungal agent, but its application in the topical treatment is limited due to its irritancy and volatility. It was aimed to develop more efficient, chitosan-incrusted MVO microspheres with reduced volatility and lesser irritancy and to dispense it in the form of ointment. Simple coacervation technique was employed to microencapsulate MVO in chitosan matrix. Morphological properties and polymer cross-linking were characterized by scanning electron microscopy and differential scanning calorimetry, respectively. Optimization was carried out on the basis of entrapment efficiency (EE) using response surface methodology. Well-designed microspheres having smooth surface and spherical shape were observed. EE (81.20\%) of optimum batch (R21) was found at $1.62 \% w / v$ of cross-linker, 5.4:5 of MVO to chitosan ratio and at $1000 \mathrm{rpm}$. R21 showed $69.38 \pm 1.29 \%$ in vitro MVO release in $12 \mathrm{~h}$ and $96.92 \%$ retention of MVO in microspheres even after 8 week. Ointments of PEG 4000 and PEG 400 comprising MVO (F1) and R21 (F2) were developed separately. F2 showed comparatively broader zone of growth inhibition $(13.33 \pm 1.76-18.67 \pm 0.88 \mathrm{~mm}$ ) and less irritancy (PII 0.5833 , irritation barely perceptible) than that of F1. F2 was able to avoid the direct contact of mild irritant MVO with the skin and to reduce its rapid volatility. Controlled release of MVO helped in lengthening the duration of availability of MVO in agar media and hence improved its therapeutic efficacy. In conclusion, a stable and non-irritant formulation with improved therapeutic potential was developed.
\end{abstract}

KEY WORDS: anti-fungal; Candida; essential oil; microspheres; primary irritation index.

\section{INTRODUCTION}

The frequency of mucosal and cutaneous fungal infections has dramatically increased worldwide, and candidiasis is most frequent among them. Candida species are major human opportunistic fungal pathogens that cause both mucosal and deep tissue infections $(1,2)$. Research in the past decades has led many natural compounds, which are effective against Candida. Essential oils, isolated from plants, have

Electronic supplementary material The online version of this article (doi:10.1208/s12249-015-0378-x) contains supplementary material, which is available to authorized users.

\footnotetext{
${ }^{1}$ Botany and Pharmacognosy Department, CSIR-Central Institute of Medicinal and Aromatic Plants, P.O. CIMAP, Lucknow, 226015, India.

${ }^{2}$ Molecular Bioprospection Department, CSIR-Central Institute of Medicinal and Aromatic Plants, P.O. CIMAP, Lucknow, 226015, India.

${ }^{3}$ To whom correspondence should be addressed. (e-mail: np.yadav@cimap.res.in; npyadav@gmail.com)

ABBREVIATIONS: MVO, Mentha spicata L. var. viridis oil; \%EE, Entrapment efficiency; RSM, Response surface methodology; CCD, Central composite design; SEM, Scanning electron microscopy; DSC, Differential scanning calorimetry; PII, Primary irritation index; ZGI, Zone of growth inhibition; PEG, Polyethylene glycol.
}

been of particular interest showing potent biological activity (3). In this perspective, Mentha spicata L. var. viridis (family: Lamiaceae) essential oil (MVO) has shown strong activity against most human fungal pathogens including Candida species (4). Therefore, it might be a good therapeutic alternative for candidiasis, but its irritant nature restricts pharmaceutical application, while rapid volatilization reduces the term of effectiveness.

To overcome the above limitations, MVO was aimed to be encapsulated in polymer matrix. Microencapsulation is one of the most effective methods to reduce irritancy and losses by evaporation (5). In this method, the wall-forming polymer plays an important role as it is responsible for the protection of the encapsulated essential oil (6). Therefore, chitosan, a natural cationic linear polysaccharide composed of randomly distributed $\beta$-(1-4)-linked D-glucosamine (deactylated unit) and $N$-acetyl-D-glucosamine (acetylated unit), was selected as it is widely used in topical dressing due to haemostatic and healing potential. The presence of umpteen number of 2-amino-2-deoxyglucose unit permits easy diffusion of polymer into the solution (7). Chitosan possesses varied biological properties such as wound healing, antibacterial, antifungal, hemostatic anticoagulant, and bacteriostatic properties and also found to be safe, biocompatible, biodegradable and bioadhesive $(5,8)$. The bioadhesive and cationic nature of chitosan is due to free amino groups which favour its 
interaction with the skin surface (9). Furthermore, after getting interacted with the skin protein, it breaks up the tight junction among the cells and increases the permeability of the stratum corneum and thus permits the penetration of active moiety into the underlying layer of epidermis (7). Also, as a wall-forming material, it helps in the protection of drug from the environmental damage and delivers the drug in controlled and sustained manner. On account of the topical drug delivery, chitosan is a suitable polymer due to its versatile biological, mechanical, physicochemical and functional properties $(8,10,11)$.

To our knowledge, chitosan has not been explored to reduce the irritation and volatilization of MVO and thus improving the therapeutic potential. Therefore, the present investigation was undertaken to explore the role of the chitosan microspheres in the safe and effective delivery of MVO using the concept of microencapsulation.

\section{MATERIALS AND METHODS}

\section{Materials}

M. spicata L. var. viridis oil (MVO) was obtained from the Department of Process Chemistry and Chemical Engineering of CSIR-CIMAP, Lucknow. Chitosan (medium molecular weight) was purchased from Sigma-Aldrich Pvt. Ltd., New Delhi, India. Sodium hydroxide pellets were procured from CDH Laboratory Limited, New Delhi. Acetic acid and lactic acid (98\%) were purchased from Thomas Baker Pvt. Ltd, Mumbai. Polyethyleneglycol-4000, Polyethyleneglycol400, propyl paraben and methyl paraben were purchased from Himedia Laboratories Pvt. Ltd., Mumbai, India. All other chemicals were of analytical grade and were used as received. Cultures of pathogenic species of Candida were obtained from CSIR-Institute of Microbial Technology, Chandigarh, India.

\section{Methods}

\section{Measurement}

Calibration curve was plotted by using the methodology reported elsewhere (12). A known concentration of MVO in ethanol/phosphate buffer $(1: 9 v / v)$ was scanned between 200 and $400 \mathrm{~nm}$ using UV-visible spectrophotometer (UV 1800 Shimadzu, Kyoto, Japan). The absorption maxima were found to be at $223 \mathrm{~nm}$. Consequently, the stock solution was prepared by dissolving $10 \mathrm{ml}$ of MVO in $10 \mathrm{ml}$ of ethanol/phosphate buffer $(1: 9 v / v)$ followed by the preparation of various dilutions in the range of 10 $50 \mu \mathrm{g} / \mathrm{ml}$. Absorption of the dilutions was taken at $223 \mathrm{~nm}$, and the calibration curve was plotted to assess transmittance of light through the samples.

\section{Experimental Design}

Central composite design (CCD) of response surface methodology (RSM) was employed to produce controlled release microspheres of MVO using a polynomial equation with the help of Design-expert ${ }^{\circledR}$ 8.0.7.1 software (Trial version; Stat-Ease Inc., USA). In this design, the individual effect of three independent variables, namely emulsification stirring rate $(\mathrm{X} 1 ; \mathrm{rpm})$, the amount of sodium hydroxide $(\mathrm{X} 2$; percentage $w / v)$ and MVO-to-chitosan ratio $(\mathrm{X} 3 ; w / w)$ were analyzed on $\% \mathrm{EE}(Y)$. The lower and higher value of polymer concentrations, cross-linker concentrations and drug-polymer ratios were selected on the basis of preliminary experimental results. The range of the actual and the coded values for the independent factors and their possible combinations as generated by the software is given in Table I. The least square regression model was fitted to the responses taken from the experimental data to define the optimization process of $\% \mathrm{EE}$. The regression model coefficient for the \%EE of MVO was evaluated using a generalized polynomial Eq. 1 as generated by the software in terms of linear, quadratic and cross-factors (13).

$Y=\beta_{0}+\sum_{i=1}^{3} \beta_{i} X_{i}+\sum_{j=1}^{3} \beta_{i i} X_{1}^{2}+\sum_{i=1}^{3} \sum_{j-i+1}^{2} \beta_{i j} X_{i} X_{j}$

Where $Y$ is the level of the measured response; $\beta_{0}, \beta_{i}, \beta_{i i}$ and $\beta_{i j}$ are regression coefficients for intercept, linear, quadratic and cross-factor coefficients, respectively; $X_{i}$ and $X_{j}$ are coded independent variables; and $X_{i} X_{j}$ is the interaction between them.

\section{Encapsulation Procedure}

Simple coacervation technique was employed to encapsulate MVO as reported by Hsieh et al. 2006 (5) with slight modification. Briefly, MVO was stained with yellow colour oil dye for tracing purpose. MVO-to-chitosan ratio was $1: 1 \mathrm{w} / \mathrm{w}$. Chitosan was solubilized in $1 \% \mathrm{v} / \mathrm{v}$ acetic acid and emulsified with MVO using homogenizer (EUROSTAR IKA® power control visc) at $1000 \mathrm{rpm}$ for $20 \mathrm{~min}$. The whole system was assembled in ice bath to minimize the risk of volatilization at higher speed. The emulsion was sprinkled in $1.5 \% \mathrm{w} / \mathrm{v}$ sodium hydroxide solution and kept as such for $60 \mathrm{~min}$ with gentle stirring. Formed microspheres were washed twice with distilled water, filtered and dried at room temperature for $3 \mathrm{~h}$. The resultant microspheres were dried in desiccator.

\section{Entrapment Efficiency}

The entrapment efficiency ( $\% \mathrm{EE})$ was determined as per the methodology reported by Maji et al. (14). An accurately weighed $(10 \mathrm{mg})$ microspheres fabricated in different conditions (Table I) were added in a conical flask containing $10 \mathrm{ml}$ of ethanol/phosphate buffer $(1: 9 v / v)$ solution and continuously stirred at $700 \mathrm{rpm}$ for $1 \mathrm{~h}$. To avoid the oil evaporation due to high temperature and stirring, the flask was assembled on ice bath. The content of each beaker was centrifuged, and supernatant was analysed at $223 \mathrm{~nm}(12,15,16)$. Each experiment was performed in triplicate, and \%EE was calculated as per Eq. 2.

$\mathrm{EE}(\%)=\left(\frac{E \mathrm{a}}{E \mathrm{t}}\right) \times 100$

Where $E_{\mathrm{a}}$ and $E_{\mathrm{t}}$ are the actual and theoretical amount of MVO encapsulated in chitosan polymer respectively. 
Table I. Central composite design for the optimization of entrapment efficiency (\%) along with coded and actual values of the independent variables

\begin{tabular}{|c|c|c|c|c|c|c|c|}
\hline \multirow[t]{2}{*}{ Experimental run } & \multicolumn{3}{|c|}{ Coded variables } & \multicolumn{3}{|c|}{ Process variables } & \multirow{2}{*}{$\begin{array}{l}\text { Response } \\
\text { Y }\end{array}$} \\
\hline & $\mathrm{X} 1$ & $\mathrm{X} 2$ & $\mathrm{X} 3$ & $\mathrm{X} 1$ & $\mathrm{X} 2$ & $\mathrm{X} 3$ & \\
\hline $\mathrm{R} 1$ & -1 & -1 & -1 & 500 & 1.0 & $1: 2$ & 68.22 \\
\hline R2 & 1 & -1 & -1 & 1500 & 1.0 & $1: 2$ & 69.01 \\
\hline R3 & -1 & 1 & -1 & 500 & 2.0 & $1: 2$ & 71.73 \\
\hline $\mathrm{R} 4$ & 1 & 1 & -1 & 1500 & 2.0 & $1: 2$ & 71.95 \\
\hline R5 & -1 & -1 & 1 & 500 & 1.0 & $3: 2$ & 74.23 \\
\hline R6 & 1 & -1 & 1 & 1500 & 1.0 & $3: 2$ & 75.1 \\
\hline R7 & -1 & 1 & 1 & 500 & 2.0 & $3: 2$ & 77.09 \\
\hline $\mathrm{R} 8$ & 1 & 1 & 1 & 1500 & 2.0 & $3: 2$ & 78.22 \\
\hline R9 & -1.68 & 0 & 0 & 160 & 1.5 & $1: 1$ & 77.07 \\
\hline R10 & 1.68 & 0 & 0 & 1840 & 1.5 & $1: 1$ & 79.44 \\
\hline R11 & 0 & -1.68 & 0 & 1000 & 0.66 & $1: 1$ & 70.18 \\
\hline R12 & 0 & 1.68 & 0 & 1000 & 2.34 & $1: 1$ & 77.32 \\
\hline $\mathrm{R} 13$ & 0 & 0 & -1.68 & 1000 & 1.5 & $4: 25$ & 65.12 \\
\hline $\mathrm{R} 14$ & 0 & 0 & 1.68 & 1000 & 1.5 & $46: 25$ & 80.02 \\
\hline $\mathrm{R} 15$ & 0 & 0 & 0 & 1000 & 1.5 & $1: 1$ & 81.68 \\
\hline R16 & 0 & 0 & 0 & 1000 & 1.5 & $1: 1$ & 81.74 \\
\hline R17 & 0 & 0 & 0 & 1000 & 1.5 & $1: 1$ & 80.57 \\
\hline R18 & 0 & 0 & 0 & 1000 & 1.5 & $1: 1$ & 80.52 \\
\hline R19 & 0 & 0 & 0 & 1000 & 1.5 & $1: 1$ & 80.05 \\
\hline R20 & 0 & 0 & 0 & 1000 & 1.5 & $1: 1$ & 80.02 \\
\hline
\end{tabular}

$X 1$ Stirring rate for emulsification (rpm), $X 2$ concentration of sodium hydroxide (\%), X3 MVO-to-chitosan ratio, $Y$ Entrapment efficiency (\%)

\section{Loading Efficiency}

Loading efficiency of optimized formulation was calculated as per Eq. 3:

$\%$ Loading efficiency $=\frac{\text { weight of MVO in microspheres }}{\text { weight of microspheres }} \times 100(3)$

\section{Particle Size Measurements}

Particle size analysis of microspheres was performed using compound microscope (LEICA ICC50 HO). Images were captured and processed by Image Leica application suite software. The slide containing microspheres was mounted on the stage of the microscope. Images of microspheres were captured using a CCD camera. The diameter of at least 130 particles was measured via image analysis using the Image LAS EZ software. The median of particle size was reported as the particle size of microspheres (17).

\section{In Vitro Release Study}

The in vitro MVO releasing property of microspheres was evaluated under the maximal yield condition of microencapsulation using modified USP dissolution apparatus type I (Electrolab, Dissolution tester, EDT-08Lx) assembled with 40 mesh size basket and $200 \mathrm{ml}$ capacity flasks. Finally, a known volume of ethanol/phosphate buffer solution $(1: 9 v / v$, $\mathrm{pH}$ 7.4) was poured into the flask, and the assembly was set at constant stirring (50 RPM) and temperature $\left(37 \pm 0.5^{\circ} \mathrm{C}\right)(18)$. At a predetermined interval, i.e. after 0, 15, 30, 60, 120, 180, 240, 480 and $720 \mathrm{~min}, 1 \mathrm{ml}$ sample was collected followed by the replenishment with the same volume of fresh and preheated receptor medium solvent at each sampling interval. The collected samples were analyzed by UVsectrophotometer at $223 \mathrm{~nm}$ after appropriate dilution. The experiment was performed in triplicate $(16,19)$.

\section{Characterization Parameters}

Scanning Electron Microscopy. The surface morphology of the optimized microsphere was studied by scanning electron microscopy (SEM) (430 LEO, Carl Zeiss, Germany, UK) using polaron sputter coater and gold platinum alloy as a coating material. The acceleration voltage during observation was $20 \mathrm{kV}$. The microspheres were mounted on a double-sided adhesive tape stuck to a gold coated (thickness $\sim 250 \AA$ ) stub. Images were taken randomly at $\times 100$ magnification (5).

Fourier Transform Infra-Red Spectroscopy. Fourier transform infra-red (FTIR) spectra of MVO, chitosan and optimized microspheres of MVO (R16) were obtained by using Perkin Elmer FT-IR Spectrum BX Spectrophotometer, PerkinElmer Ltd., Waltham, U.S.A. Potassium bromide $(\mathrm{KBr})$ pellets were prepared by mixing $2 \mathrm{mg}$ of sample and $200 \mathrm{mg}$ of $\mathrm{KBr}$ followed by compression. The scanning range and the resolution were 650 to 4000 and $1 \mathrm{~cm}^{-1}$, respectively. For FT-IR spectra of MVO, thin film was applied directly on $\mathrm{KBr}$ plate and scanned between 600 and $4000 \mathrm{~cm}^{-1}$ (6).

Differential Scanning Calorimetry Analysis. The differential scanning calorimetry (DSC) curves of chitosan and optimized microspheres were obtained using Diamond DSC (Perkin Elmer, Wellesley, USA). An accurately weighed sample $(3.5 \mathrm{mg})$ was heated in hermetically sealed aluminium pans over a temperature range of $40-450^{\circ} \mathrm{C}$ at a constant rate 
$\left(20^{\circ} \mathrm{C} / \mathrm{min}\right.$ ) under nitrogen gas (at $30 \mathrm{ml} / \mathrm{min}$ flow rate). The obtained thermo grams were analysed and interpreted as reported elsewhere (20).

\section{Validation of Experimental Design}

To validate the experimental design, an extra check point formulation was prepared (R21) by taking the values of independent variable (as suggested by the software). The experimental value of the entrapment efficiency of R21 was determined and compared with the predicted value (21).

\section{Incorporation of Microspheres into Ointment Bases}

Accurately weighed quantity of MVO $(2 \% w / w)$ and the optimized microspheres equivalent to MVO were incorporated separately into an ointment base comprising of polyethyleneglycol-4000, polyethyleneglycol-400, propyl paraben as oil phase and methyl paraben in the aqueous phase [formula for ointment base given in Supplementary Table ST]. Aqueous and oil phase were stirred and heated up to $58-60^{\circ} \mathrm{C}$ separately. The aqueous phase was poured and emulsified into the oil phase for $15 \mathrm{~min}$. The ointment was allowed to cool followed by the addition of $2 \% w / w$ MVO (F1) and optimized microspheres corresponding to $2 \%$ of MVO (F2) separately, with continuous stirring until the mixture congealed.

\section{Drug Content and Content Uniformity}

The MVO content of the prepared ointment was carried out by dissolving accurately weighed quantity of ointment equivalent to $10 \mathrm{mg}$ of the MVO in $100 \mathrm{ml}$ volumetric flask and volume was made up to $100 \mathrm{ml}$ with ethanol/phosphate buffer $(1: 9 v / v)$ solution. The content was centrifuged and supernatant was analysed at $223 \mathrm{~nm}$. The MVO content was determined from the calibration curve. The tests were carried out in triplicate. Similarly, the content uniformity was determined by analysing MVO concentration in ointment taken from three different layers of the container.

\section{Antifungal Activity}

The quantitative antifungal activity of MVO, MVO in ointment (F1) and optimized microencapsulated MVO in ointment (F2) were performed against selected pathogenic strains of Candida using agar well diffusion assay $(22,23)$. About $100 \mu$ l of the inoculum suspension of each test organism was distributed evenly over the surface of sabouraud dextrose agar plate. Two well each of $7 \mathrm{~mm}$ diameter were bored in a single plate and filled with F1 and F2 (amount equivalent to $4 \mu \mathrm{l}$ of MVO). A disk soaked in $4 \mu \mathrm{l}$ of MVO was also placed on the same plate. The plates were incubated for 3 days at $37^{\circ} \mathrm{C}$ (LT$\mathrm{X}$, Labtherm, Kuhner). Experiments were performed in triplicate, and zone of growth inhibitions (ZGI) were measured in millimetre ( $\mathrm{mm})(18)$.

Skin Irritation Study

Experimental Animals. Adult New Zealand white rabbits of either sex, having body weight $\sim 2.5 \mathrm{~kg}$, were received from Animal House of CSIR-CIMAP, Lucknow, India. The animals were acclimatized to the experimental environment for 7 days before commencing the experiment $\left(22 \pm 5^{\circ} \mathrm{C}\right.$ with $55 \pm 5 \% \mathrm{RH}$ and $12 \mathrm{~h}$ dark/light cycle). The animals were reused after a wash period of 15 days. Animals were provided ad libitum access to a commercial rabbit diet (Dayal Industries, Lucknow) and drinking water. The experiment was carried out in accordance of OECD test guideline no. 404 (24). The protocol (Reg. No. 400/01/AB/CPCSEA, AH-2012-01) was duly approved by the Institutional Animal Ethics Committee (IAEC) under CPCSEA (Govt. of India) guidelines.

Experimental Protocol. The back of each rabbit $(n=6)$ was clipped free of fur with curved scissor before $24 \mathrm{~h}$ of the application of the sample. The clipped area of skin was divided into two test sites of 1 square inch each. Normal saline was chosen as vehicle control and lactic acid (98\% in distilled water) as positive control (25). Rabbits were selected randomly and single test sample (MVO, ointment base, F1, F2 and lactic acid 98\%) was applied at a time on one test site of the animal against vehicle control following a prescribed wash period of 15 days for the subsequent treatment. All the sites were covered with gauze, and the back of the rabbit was wrapped with a non-occlusive bandage. After $4 \mathrm{~h}$, the bandage was removed, sites were macropathologically examined for skin irritation and the observation was repeated after 24, 48 and $72 \mathrm{~h}$. Skin reactions are graded separately for erythema and edema, each time on a 0-4 grading scale. The primary irritation index (PII) was calculated as the arithmetic mean of erythema/edema scores of the six animals, i.e. of the six patches with the same test material. Test materials were categorized on the basis of PII values mentioned under OECD Test guideline no. 404. Comparison between the mean values of PII of the experimental groups was made by one-way analysis of variance (ANOVA) followed by Tukey's post hoc test using GraphPad® Prism, Version 5.01 (GraphPad Software. Inc., USA). The statistical significance of differences was accepted at $p \leq 0.05$ (26).

\section{Stability of MVO Microspheres}

The optimized microspheres were analysed for percentage MVO retention during an 8-week storage at 27 $\pm 2{ }^{\circ} \mathrm{C}$. The percentage retention of MVO was calculated using:

$$
\begin{aligned}
& \% \text { Retention } o \text { AVO } \\
& =\frac{\text { MVO at }{ }^{\prime} X^{\prime} \text { day of storage time }\left(A_{t}\right)}{\text { MVO at ' } 0^{\prime} \text { day of storage time }(A o)} \times 100
\end{aligned}
$$

Stability of encapsulated MVO was fitted in first order kinetic model during storage.

$$
\frac{A_{t}}{A o}=\exp (-k t)
$$

Where $A_{o}$ and $A_{t}$ are the content of MVO immediately after encapsulation and after time $t$, respectively. A log of percent 
retention of MVO vs. time was plotted to obtain the rate constant $(\mathrm{k})$ as the slope of the graph from which the halflife $\left(t_{1 / 2}\right)$ of MVO (the time required for $50 \%$ reduction in MVO content) was calculated using $0.693 / \mathrm{k}$ equation (27).

\section{RESULTS}

\section{Optimization of MVO Microencapsulation}

Based on the preliminary experiments, a simplified 20 experimental set of three independent variables (Table I), namely emulsification stirring rate, cross-linker concentration and MVO-to-chitosan ratio, was obtained using CCD. To determine the optimal condition for microencapsulation and to establish the relationship between the entrapment efficiency $(\% \mathrm{EE})$ and selected variables, it is required to test the significance and adequacy of the model using analysis of variance (ANOVA) through a joint test of three parameters (Table II). Thus, experimentally determined responses (entrapment efficiency) of all the 20 batches were used to get regression coefficient and polynomial equation of fit (in terms of coded values) which is as follows:

$$
\begin{aligned}
\% \mathrm{EE}= & 80.81+0.51 X 1+1.79 X 2+3.57 X 3-0.039 \\
& X 1 X 2+0.12 X 1 X 3-0.059 X 2 X 3-1.16 \\
& X 1^{2}-2.76 X 2^{2}-3.17 X 3^{2}
\end{aligned}
$$

Where $\mathrm{X} 1, \mathrm{X} 2$ and $\mathrm{X} 3$ are the coded values for emulsification stirring rate, cross-linker concentration and MVO-to-chitosan ratio, respectively.

Measured \%EE of 20 different batches was found to be in the range of $65.12-81.74 \%$. Corresponding to experimentally determined \%EE, 81.74 (Table I), the predicted \%EE was found to be $81.195 \%$ under optimum condition (1.62 $\mathrm{mg} / \mathrm{ml}$ for cross-linker and 5.39:5 for MVO-to-chitosan ratio at zero level of stirring speed as suggested by the software), which is nearly similar to the experimental value (Fig. 1a). As evident from the Fisher's $F$ test (F model=29.99), the analysis of variance of the regression model was found highly significant, and the goodness of fit was confirmed by the determination of regression coefficient $\left(r^{2}\right)$. In this experiment, the value of the determination coefficient indicates that $96.43 \%$ variation in the response could be explained by the model (Table II). The result represents that the regression equation was a suitable model to describe the response of the experimental parameters.

The 2D contour plots represent the interaction between the variables and to locate the optimum level of each variable for maximum response. Each contour plot for \% EE represents the different combinations of two test variables at one time while keeping the other variable at their respective zero level. There were six pairs of contour plots in this work and three typical ones are shown in Fig. 1b. The interactions between the variables can be inferred from the shapes of the contour plots. Circular contour plots indicate that the interactions between the variables are negligible while the elliptical ones indicate the evidence of interactions (28). The elliptical 2D contour plot depicted in Fig. 1b confirmed that interaction occured between stirring rate and drug polymer ratio (Fig. 1b (ii)) compared to other plots which are circular (Fig. 1b (i), (iii)).

\section{Particle Size}

Average particle size of 130 microspheres was found to be $835.82 \pm 90.48 \mu \mathrm{m}$ which was in accordance with the SEM analysis.

\section{In Vitro MVO Release}

figure 2 represents the release profile of the optimized batch (in triplicate) which was found to be almost identical to each other and exhibited the minimum burst effect. All the microspheres have shown the continuous release of the con-

\begin{tabular}{|c|c|c|c|c|}
\hline Factor & Coefficient estimate & Standard error & $\mathrm{P}$ value Prob $>\mathrm{F}$ & $F$ value \\
\hline Constant & 80.80551 & 0.532031 & $<0.0001$ & 29.98729 \\
\hline \multicolumn{5}{|l|}{ Linear } \\
\hline $\mathrm{X} 1$ & 0.512259 & 0.35299 & 0.1774 & 2.105979 \\
\hline$X 2$ & 1.789431 & 0.35299 & 0.0005 & 25.69831 \\
\hline $\mathrm{X} 3$ & 3.572471 & 0.35299 & $<0.0001$ & 102.4264 \\
\hline \multicolumn{5}{|l|}{ Interactions } \\
\hline $\mathrm{X} 1 \mathrm{X} 2$ & -0.03875 & 0.461204 & 0.9347 & 0.007059 \\
\hline $\mathrm{X} 1 \mathrm{X} 3$ & 0.12375 & 0.461204 & 0.7939 & 0.071995 \\
\hline X2 X3 & -0.05875 & 0.461204 & 0.9012 & 0.016227 \\
\hline \multicolumn{5}{|l|}{ Quadratic } \\
\hline $\mathrm{X} 1^{2}$ & -1.16251 & 0.343626 & 0.0070 & 11.44509 \\
\hline $\mathrm{X} 2^{2}$ & -2.75527 & 0.343626 & $<0.0001$ & 64.29157 \\
\hline $\mathrm{X} 3^{2}$ & -3.17246 & 0.343626 & $<0.0001$ & 85.2352 \\
\hline$r^{2}$ & & & & 0.9643 \\
\hline Model F value & & & & 29.99 \\
\hline Probability of F & & & & 0.0001 \\
\hline Lack of Fit $F$ value & & & & 4.76 \\
\hline
\end{tabular}
tent $(69.38 \pm 1.29 \%)$ during the $12 \mathrm{~h}$ of the study period.

Table II. ANOVA table for response surface quadratic model 


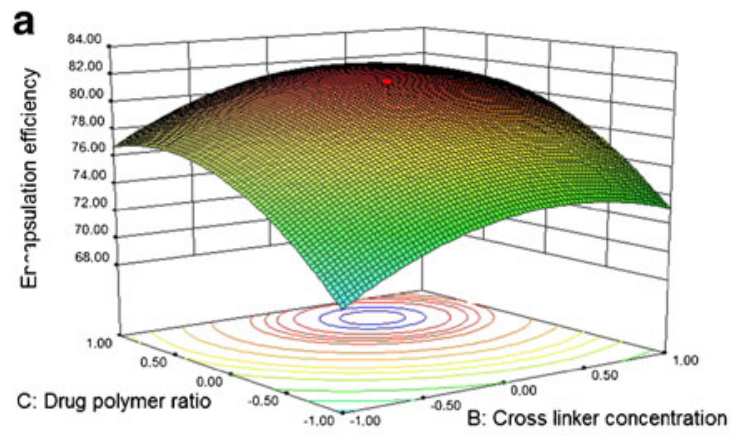

b
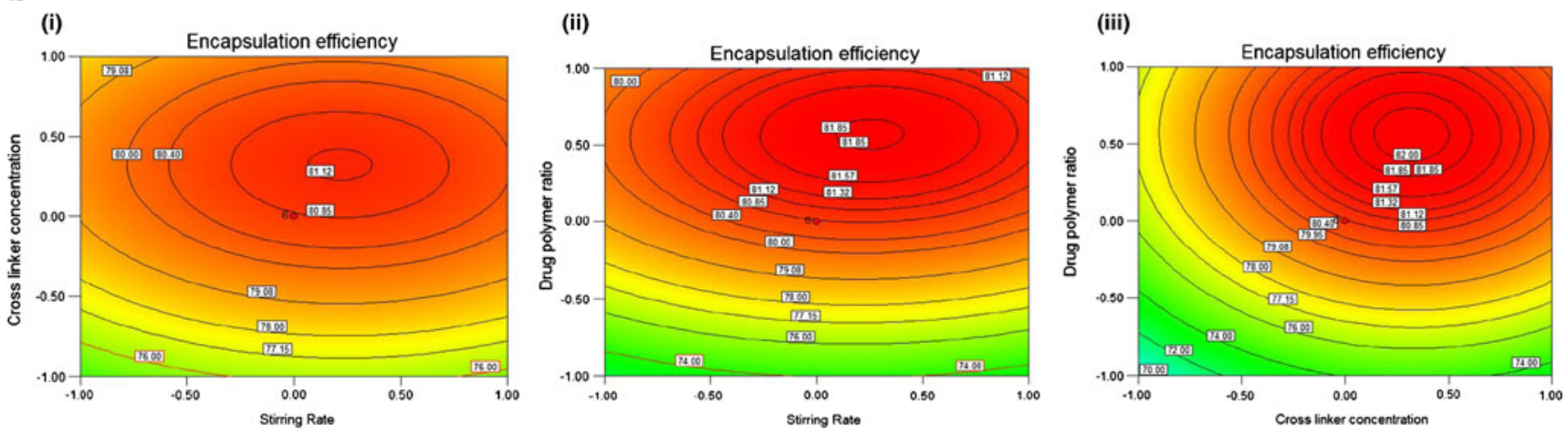

Fig. 1. Pictorial representation of experimental design (a). Response surface of EE of microencapsulated MVO shown between cross-linker concentration (X2) and MVO-to-chitosan (X3) ratio (b). 2D Contour plots between various formulation parameters as suggested by CCD $i$ interaction between stirring rate (X1) and cross-linker concentration (X2), ii interaction between stirring rate (X1) and MVO to chitosan ratio (X3) and iii interaction between cross-linker concentration (X2) and MVO-to-chitosan ratio (X3)

\section{Characterization Parameters}

\section{Scanning Electron Microscopy}

The purpose of SEM study was to obtain a topographical characterization of microspheres. SEM image showed that dried microspheres possessed spherical/oval shape with continuous and smooth surfaces (Fig. 3a).

\section{Fourier Transform Infra-Red Spectroscopy}

The MVO contains piperitenone, carvone and limonene as the major constituent (4). The FT-IR spectra of MVO (Fig. 3b (i)) indicated frequencies with methylene group twisted in vibrational modes at 670.35 and $764.40 \mathrm{~cm}^{-1}$ and out-ofplane $\mathrm{C}-\mathrm{C}-\mathrm{H}$ wagging of the di-substituent olefinic part of the molecule at $915.62 \mathrm{~cm}^{-1}$ and the symmetric $\mathrm{C}-\mathrm{H}$ bending modes of the methyl groups at 1437.67 and $1457.87 \mathrm{~cm}^{-1}$. The symmetric $\mathrm{C}=\mathrm{C}$ stretching of the ring was found at $1718.23 \mathrm{~cm}^{-1}$ whereas the corresponding vibration of the side chain was found at $1734.45 \mathrm{~cm}^{-1}$. The strongest vibration in the IR spectrum, i.e. $\mathrm{C}=\mathrm{O}$ stretching, was found at $1789.2 \mathrm{~cm}^{-1}$. For methyl group, one distinct band occurring at $2961.70 \mathrm{~cm}^{-1}$ resulted from asymmetrical stretching mode in which two $\mathrm{C}-\mathrm{H}$ bonds were extending while the third one was contracting and for methylene group asymmetrical stretching and symmetrical stretching occurred near 2928.11 and $2859.42 \mathrm{~cm}^{-1}$, respectively.

Chitosan exhibited main characteristic bands of carbonyl $(\mathrm{C}=\mathrm{O}-\mathrm{NHR})$ and amine group $\left(-\mathrm{NH}_{2}\right)$ at 1655.34 and
$1544.29 \mathrm{~cm}^{-1}$, respectively. The broad band due to the stretching vibration of $-\mathrm{NH}_{2}$ and $-\mathrm{OH}$ group was observed at 3428.4 and $3529.15 \mathrm{~cm}^{-1}$, respectively. The bands at $895.22-$ $1253.58 \mathrm{~cm}^{-1}$ were attributed to the saccharide structure of chitosan (Fig. 3b (ii)).

The spectrum of encapsulated microspheres exhibited the characteristic peaks of MVO and chitosan. The absorbance at 1544 and $1655.38 \mathrm{~cm}^{-1}$ in microsphere spectrum showed the existence of amine and carbonyl group, respectively. The broad band due to the stretching vibration of $-\mathrm{NH}_{2}$ and $-\mathrm{OH}$ group can be observed at 3400.75$3528.34 \mathrm{~cm}^{-1}$ from chitosan wall while the peak at 2925.88, 2855.36 and $1736.63 \mathrm{~cm}^{-1}$ from MVO confirmed

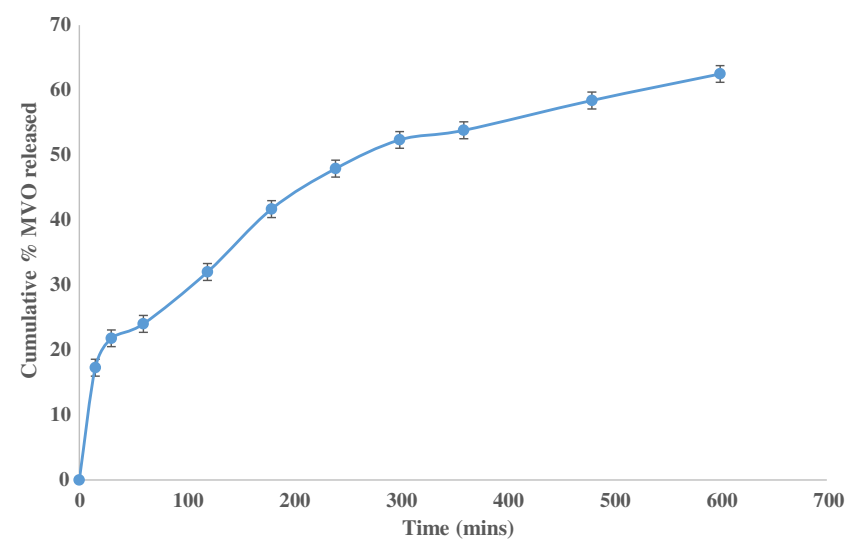

Fig. 2. Cumulative percent released of MVO from optimized microspheres in phosphate buffer ( $\mathrm{pH} 7.4)$ 
a
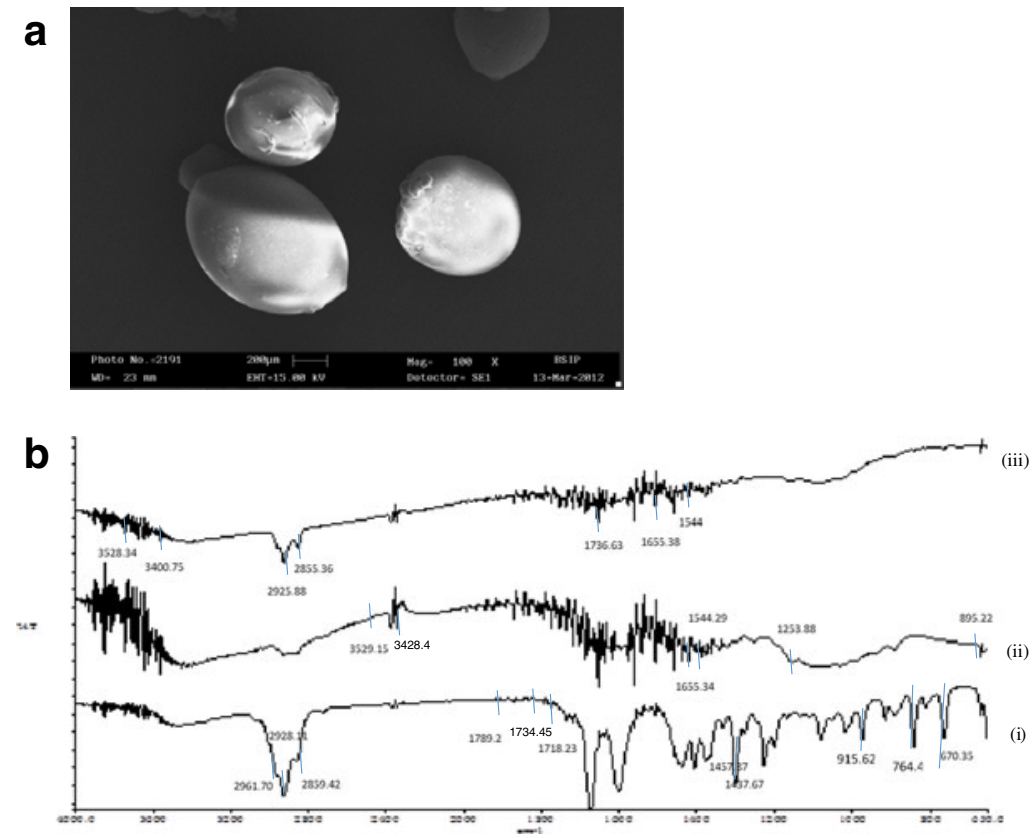

C
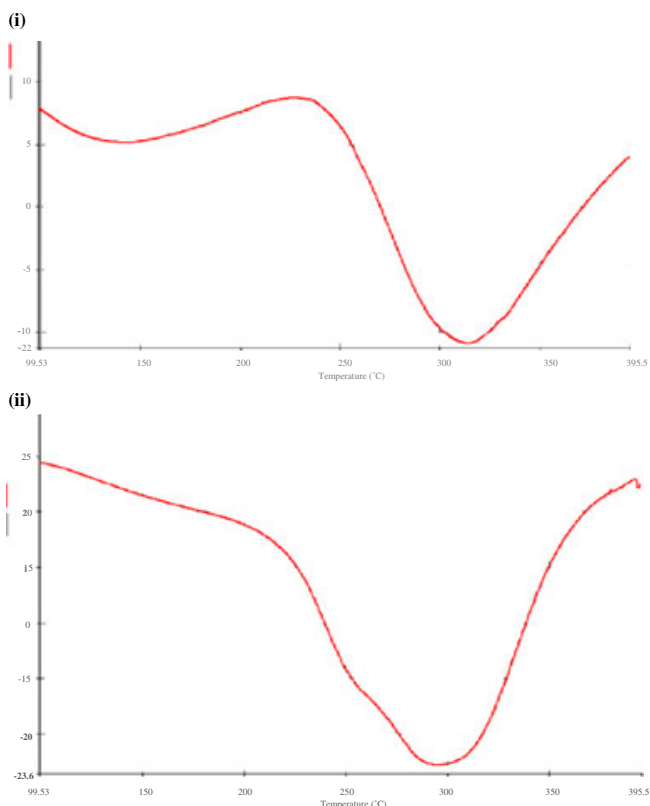

Fig. 3. Characterization parameters for microencapsulation of MVO a Scanning electron microscopy of microspheres b FT-IR spectra of $i$ MVO, ii chitosan and iii optimized microspheres $\mathbf{c}$ DSC thermograms of $i$ chitosan and ii microsphere

that there was no chemical interaction between the two (Fig. 3b(iii)). Hence, FT-IR spectrum revealed that the developed microspheres contained the chemical ingredients and functional groups of the wall material (chitosan) and the core active ingredient (essential oil).

\section{Differential Scanning Calorimetry}

The differential scanning calorimetry (DSC) thermograms of polymer and microspheres are represented in Fig. 3c. The endothermic peak of chitosan was found at $325^{\circ} \mathrm{C}$ (Fig. 3c (i)), which is corresponding to melting point of chitosan, but in case of encapsulated microspheres, the peak was shifted below $300^{\circ} \mathrm{C}$ (Fig. 3c (ii)).

\section{Validation of Experimental Design}

An extra design check point formulation (R21) was prepared, and the experimental value for \%EE was determined. The close resemblance between experimental (\%EE 81.107) and predicted values (\%EE 81.195) indicated the validity of experimental design $(P>0.01 ; \mathrm{F}$ calculated $<$ F tabulated).

\section{Drug Content and Content Uniformity}

The MVO content of ointment was found to be 95.36 $\pm 0.654 \%$. There was no significant difference observed at different layers, indicating that the method used to disperse the MVO microspheres in the ointment base was appropriate.

\section{Antifungal Activity}

Antifungal potential of MVO, F1 and F2 against selected pathogenic species of Candida is shown in Table III. A broader zone of growth inhibition (ZGI) was observed in the case of F2 $(13.33 \pm 1.76-18.67 \pm 0.88 \mathrm{~mm})$ as compared to F1 $(10.00 \pm 0.58-12.67 \pm 2.66 \mathrm{~mm})$ and pure MVO $(08.67 \pm 1.76-$ $12.00 \pm 1.16 \mathrm{~mm}$ ) while ointment base did not show any ZGI.

\section{Skin Irritation Index}

The results obtained from the primary skin irritation studies are listed in Table IV. The PII of positive control group

Table III. Antifungal activity of MVO, F1 and F2F1: MVO in ointment base; F2: Microencapsulated MVO in ointment base; values are mean $\pm \mathrm{SE}$

\begin{tabular}{|c|c|c|c|c|c|}
\hline \multirow[t]{2}{*}{ Parameters } & & \multicolumn{4}{|c|}{ Formulations } \\
\hline & & MVO & Ointment base & $\mathrm{F} 1$ & $\mathrm{~F} 2$ \\
\hline \multirow{4}{*}{$\begin{array}{l}\text { Zone of growth inhibition in } \mathrm{mm} \text { against } \\
\text { different strains of Candida }\end{array}$} & Candida albicans (ATCC 14053) & $12.00 \pm 1.16$ & 0 & $12.67 \pm 2.66$ & $18.67 \pm 0.88$ \\
\hline & Candida albicans (Clinical isolates) & $08.67 \pm 1.76$ & 0 & $10.00 \pm 0.58$ & $14.67 \pm 0.33$ \\
\hline & Candida kefyr (ATCC 204093) & $10.67 \pm 0.67$ & 0 & $11.33 \pm 1.33$ & $14.33 \pm 0.33$ \\
\hline & Candida tropicalis (ATCC 201380) & $10.33 \pm 1.20$ & 0 & $10.67 \pm 0.88$ & $13.33 \pm 1.76$ \\
\hline
\end{tabular}




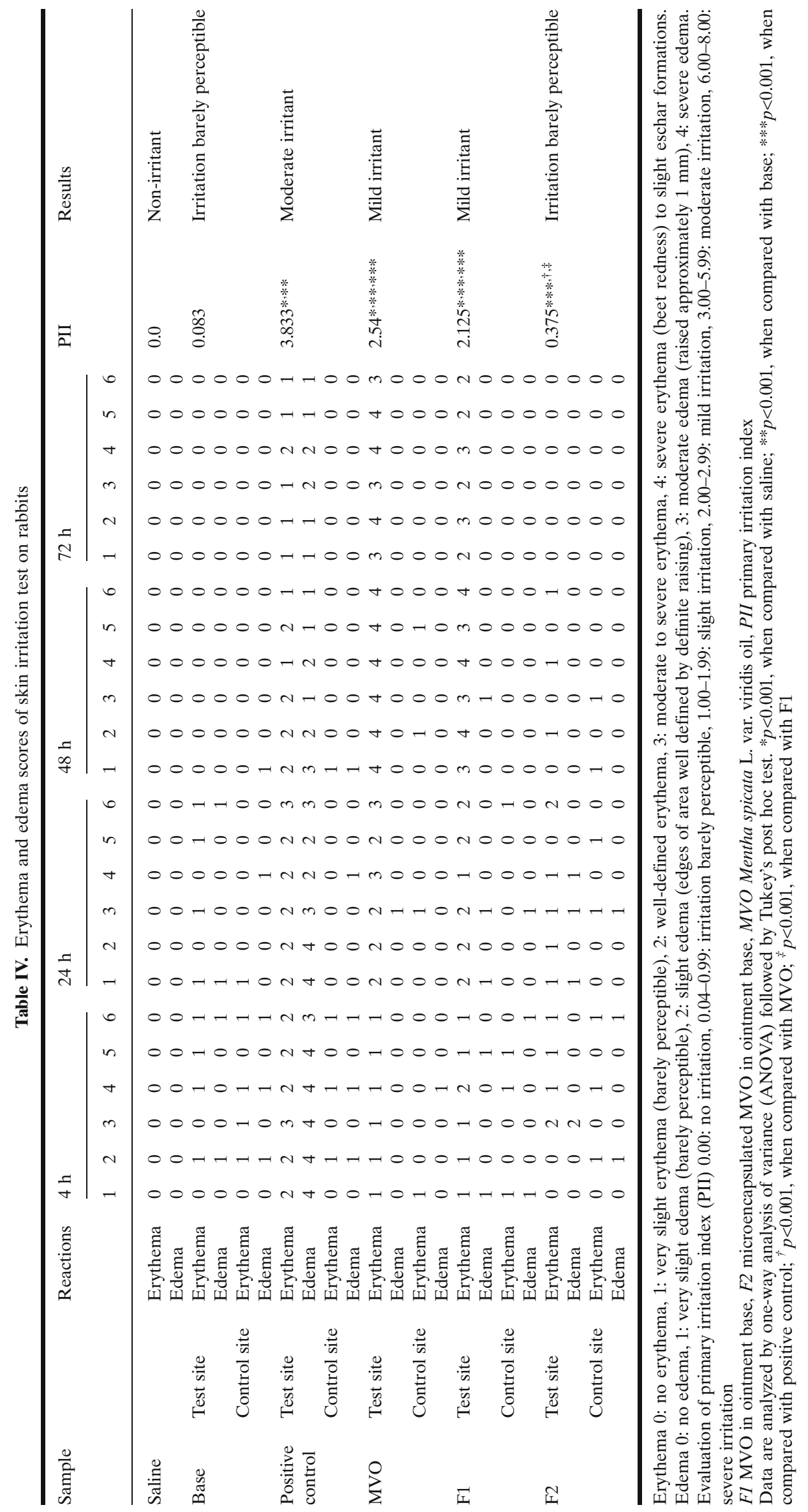


(3.833), MVO (2.541) and F1 (2.125) was found to be significantly $(p<0.001)$ different from vehicle control group $(0.0)$ and ointment base (0.083). The insignificant difference between PII of MVO and F1 indicated that both were equally irritant (mild irritant). The PII of F2 (0.375) was found to be significantly $(p<0.001)$ different from the PII of positive control group, MVO and F1 while it was insignificantly different from vehicle control group and ointment base. The PII of F1 and F2 also differed significantly $(p<0.001)$ (Fig. 4).

\section{Stability of MVO Microspheres}

Microspheres were subjected to storage stability study for a period of 8 weeks at ambient temperature $\left(27 \pm 2^{\circ} \mathrm{C}\right)$ and analyzed for percent retention of total MVO within the microspheres. A linear nature $\left(r^{2}=0.9555\right)$ of $\log$ plot of percent retention of MVO vs. storage time showed that the loss of MVO from the microspheres followed firstorder kinetics (Fig. 5). Microspheres containing MVO demonstrated significantly higher $\mathrm{t}_{1 / 2}$ (14.16 weeks) value and higher retention of MVO (96.92\%) even after 8 weeks of storage period.

\section{DISCUSSION}

Microspheres of MVO were prepared, optimized (via RSM) and evaluated successfully. Oil was found to be efficiently loaded $(52.3 \pm 0.876 \%)$ in to the microspheres which avoided the direct contact of oil with the skin and resulted in

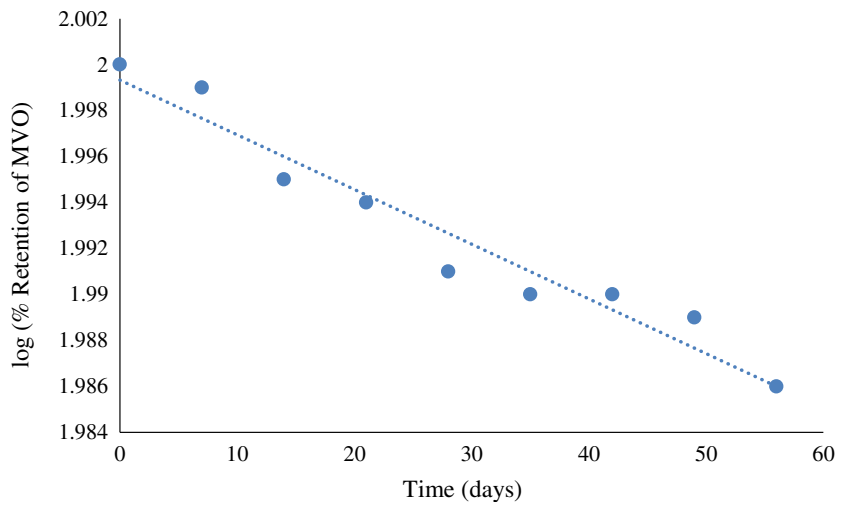

Fig. 5. Stability of encapsulated MVO measured as $\log \%$ retention of MVO vs. storage time in days

reduced irritancy. The linear and quadratic term of crosslinker concentration (X2) and MVO/chitosan ratio (X3) had significant effects on the microencapsulation efficiency, whereas the stirring rate (X1) had negligible effect on the \%EE. Therefore, $\mathrm{X} 2$ and $\mathrm{X} 3$ are the limiting factors as small variation in their concentrations would alter the \%EE to a considerable extent. The entrapment of MVO in chitosan might be due to extensive active amine and hydroxyl groups which allow subsequent modification intended for the attachment of drug (29).

The in vitro release data showed continuous release of MVO for more than $12 \mathrm{~h}$ which might be due to microencap-

\section{$4 \mathrm{~h}$}

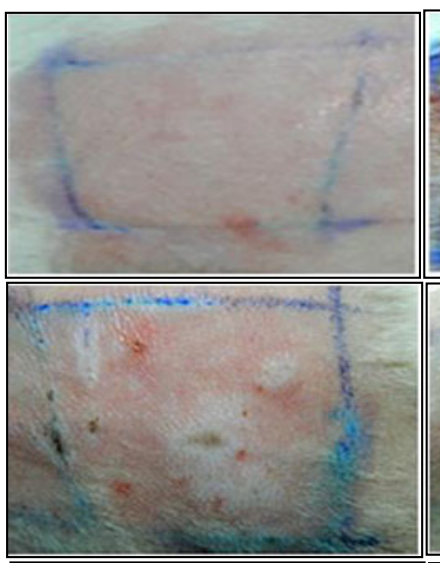

$24 \mathrm{~h}$

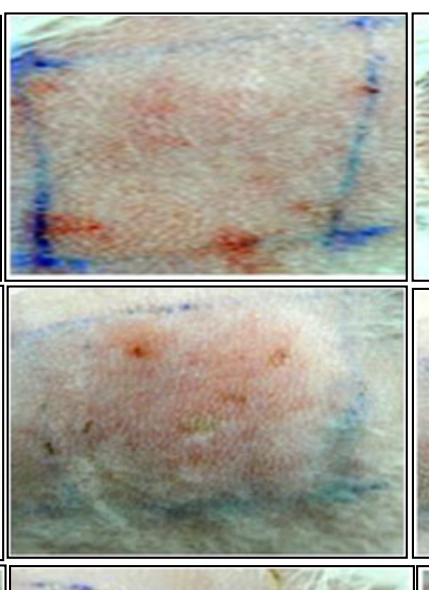

$48 \mathrm{~h}$

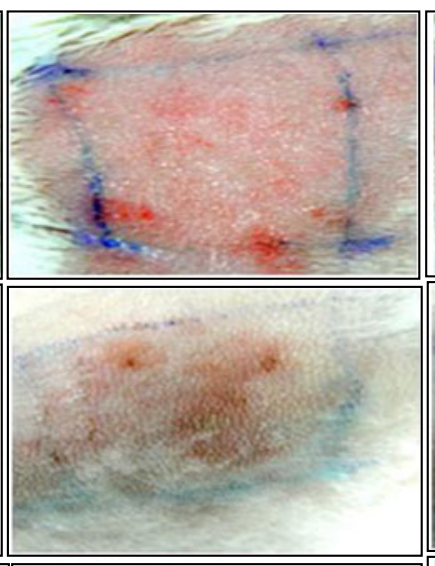

$72 \mathrm{~h}$

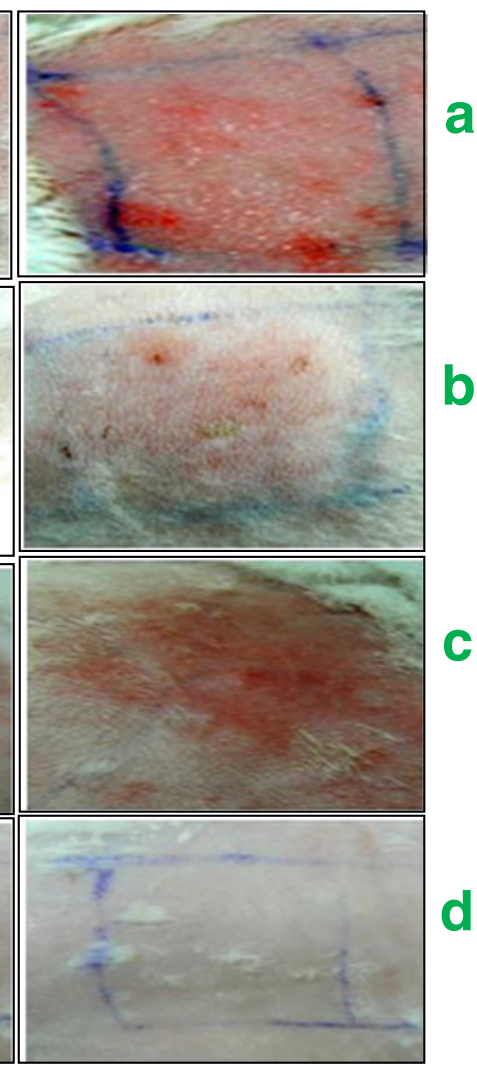

Fig. 4. Photographs of skin irritation studies carried out on New Zealand rabbits after 4, 24, 48 and 72 h, a MVO, b lactic acid (98\%), c nonmicroencapsulated oil in ointment base and $\mathbf{d}$ microencapsulated oil in ointment base 
sulation and complex formation (10). SEM, FT-IR and DSC analysis confirmed the microencapsulation of MVO in chitosan polymer. SEM showed dried, spherical/oval shape microspheres with continuous and smooth surfaces ascertaining proper cross-linking of MVO in chitosan polymer. FT-IR spectrum revealed that the developed microspheres contained the chemical ingredients and functional groups of the wall material (chitosan) and the core active ingredient (essential oil), thereby confirming some chemical interaction between chitosan and MVO. Moreover, DSC analysis showed shifting of peak below melting point of chitosan, suggesting physical interaction between MVO and chitosan, thereby confirming the microencapsulation of MVO. The antifungal activity showed broader ZGI in case of F2 which could be due to the result of reduced volatility, higher retention and prolonged release of MVO from microspheres leading to the deep penetration of MVO into the growth media. Moreover, the presence of numerous hydroxyl groups in chitosan allows it to absorb water. Interpenetration of water molecules into the microspheres results in swelling, thus gradually widening the pores of polymer network. This leads in turn to release the MVO into the surroundings resulting in good antifungal activity (30). The skin irritation study suggest the appropriateness of chitosan to diminish erythematous and edematous episodes produced by MVO as microencapsulation might have reduced its direct contact with the stratum corneum (31). Surface oil is an important factor influencing the stability of the microspheres as the surface oil can easily oxidize resulting in unacceptable off-flavors. Chitosan had lower surface oil due to uniform film formation of the microspheres which prove that chitosan has better film forming ability and consequently better retention of MVO within the microspheres.

\section{CONCLUSION}

M. spicata L. var. viridis oil (MVO) was successfully microencapsulated in chitosan polymer using simple coacervation technique. This study underlines the chitosan's ability in avoiding direct contact of MVO with the skin surface and placing the MVO formulation in the category of irritation barely perceptible. Furthermore, insignificant variation in percentage retention in microsphere, i.e. over the storage period of 8 weeks, revealed reduced volatility of MVO due to incrustation by chitosan polymer. In conclusion, the technique of microencapsulation was found to be efficient enough in developing stable, efficient and safe formulation of MVO, which can be used as an alternative to treat candidiasis.

\section{ACKNOWLEDGMENTS}

The authors are obliged to the Council of Scientific and Industrial Research (CSIR), New Delhi and Director, CSIRCentral Institute of Medicinal and Aromatic Plants, Lucknow (India) for providing financial support and infrastructure facilities under CSIR Network Project ChemBio (BSC 0203), to complete the research work. Authors are also thankful to Dr. Suaib Luqman for helping to improve the language of the manuscript.
Conflict of Interest The authors state no conflict of interest.

\section{REFERENCES}

1. Cevher E, Acma A, Sinani G, Aksu B, Zloh M, Mulazimoglu L. Bioadhesive tablets containing cyclodextrin complex of itraconazole for the treatment of vaginal candidiasis. Int $\mathbf{J} \mathrm{Biol}$ Macromol. 2014;69C:124-36.

2. Simonetti G, Santamaria AR, D’Auria FD, Mulinacci N, Innocenti M, Cecchini F, et al. Evaluation of anti-candida activity of Vitis vinifera L. seed extracts obtained from wine and table cultivars. Biomed Res Int. 2014;2014, Article ID 127021, 11 pages.

3. Dhawan S, Pal A, Ancha R, Bawankule D, Yadav N, Darokar M, et al. Efficacy of CIM 1166, a combination of compounds derived from Mentha spp. in alleviating experimental vulvovaginal candidiasis in mice. World J Microbiol Biotechnol. 2009;25(1):161-3.

4. Khanuja SPS, Kumar S, Shasany AK, Dhawan S, Darokar MP, Tripathy AK, et al. Mint plant Mentha spicata L. var. viridis christened as 'Ganga'. US Patents no. US2005/0044600 P1, 2006.

5. Hsieh WC, Chang CP, Gao YL. Controlled release properties of chitosan encapsulated volatile citronella oil microcapsules by thermal treatments. Colloid Surface B. 2006;53(2):209-14.

6. Beirão da Costa S, Duarte C, Bourbon AI, Pinheiro AC, Serra AT, Moldão Martins M, et al. Effect of the matrix system in the delivery and in vitro bioactivity of microencapsulated oregano essential oil. J Food Eng. 2012;110(2):190-9.

7. Kumar MN, Muzzarelli RA, Muzzarelli C, Sashiwa H, Domb AJ. Chitosan chemistry and pharmaceutical perspectives. Chem Rev. 2004;104(12):6017-84.

8. Dai T, Tanaka M, Huang YY, Hamblin MR. Chitosan preparations for wounds and burns: antimicrobial and wound-healing effects. Expert Rev Anti-Infect Ther. 2011;9(7):857-79.

9. Ridolfi DM, Marcato PD, Justo GZ, Cordi L, Machado D, Duran N. Chitosan-solid lipid nanoparticles as carriers for topical delivery of tretinoin. Colloid Surface B. 2012;93:36-40.

10. Muzzarelli RAA. Nanochitins and nanochitosans, paving the way to eco-friendly and energy-saving exploitation of marine resources. In: Matyjaszewski K, Möller M, editors. Polymer Science: A Comprehensive Reference. Amsterdam: Elsevier; 2012. p. 153-64.

11. Rinaudo M. Physical properties of chitosan and derivatives in sol and gel states. In: Chitosan-based systems for biopharmaceuticals. Wiley; 2012. p. 23-43.

12. Averina E, Allémann E. Encapsulation of alimentary bioactive oils of the Baikal Lake area into $\mathrm{pH}$-sensitive micro- and nanoparticles. LWT Food Sci Technol. 2013;53(1):271-7.

13. Solomon B, Sahle FF, Gebre-Mariam T, Asres K, Neubert RH. Microencapsulation of citronella oil for mosquito-repellent application: formulation and in vitro permeation studies. Eur J Pharm Biopharm. 2012;80(1):61-6.

14. Maji TK, Baruah I, Dube S, Hussain MR. Microencapsulation of Zanthoxylum limonella oil (ZLO) in glutaraldehyde crosslinked gelatin for mosquito repellent application. Bioresour Technol. 2007;98(4):840-4.

15. Chung SK, Seo JY, Lim JH, Park HH, Yea MJ, Park HJ. Microencapsulation of essential oil for insect repellent in food packaging system. J Food Sci. 2013;78(5):E709-14.

16. Songkro S, Hayook N, Jaisawang J, Maneenuan D, Chuchome T, Kaewnopparat N. Investigation of inclusion complexes of citronella oil, citronellal and citronellol with $\beta$-cyclodextrin for mosquito repellent. J Incl Phenom Macrocycl Chem. 2012;72(34):339-55.

17. Bagheri KS, Mirzadeh H, Etrati-Khosroshahi M, Shokrgozar MA. Particle size modeling and morphology study of chitosan/ gelatin/nanohydroxyapatite nanocomposite microspheres for 
bone tissue engineering. J Biomed Mater Res A. 2013;101(6):1758-67.

18. Rai VK, Yadav NP, Sinha P, Mishra N, Luqman S, Dwivedi H, et al. Development of cellulosic polymer based gel of novel ternary mixture of miconazole nitrate for buccal delivery. Carbohydr Polym. 2014;103:126-33.

19. Yenilmez E, Başaran E, Yazan Y. Release characteristics of vitamin $\mathrm{E}$ incorporated chitosan microspheres and in vitro-in vivo evaluation for topical application. Carbohydr Polym. 2011;84(2):807-11.

20. Bai C, Peng H, Xiong H, Liu Y, Zhao L, Xiao X. Carboxymethylchitosan-coated proliposomes containing coix seed oil: characterisation, stability and in vitro release evaluation. Food Chem. 2011;129(4):1695-702.

21. Arya P, Pathak K. Assessing the viability of microsponges as gastro retentive drug delivery system of curcumin: optimization and pharmacokinetics. Int J Pharm. 2014;460(1-2):1-12.

22. Carović-Stanko K, Orlić S, Politeo O, Strikić F, Kolak I, Milos M, et al. Composition and antibacterial activities of essential oils of seven Ocimum taxa. Food Chem. 2010;119(1):196-201.

23. Zhang H, Cui Y, Zhu S, Feng F, Zheng X. Characterization and antimicrobial activity of a pharmaceutical microemulsion. Int $\mathrm{J}$ Pharm. 2010;395(1-2):154-60.

24. OECD, Test No. 404: Acute dermal irritation/corrosion. OECD guidelines for the testing of chemicals, section 4, OECD Publishing, Paris (2002).

25. Kojic Z, Stojanovic D, Popadic S, Jokanovic M, Janackovic D. The irritative property of alpha-tricalcium phosphate to the rabbit skin. Gen Physiol Biophys. 2009;28 Spec No:168-73.
26. Yadav NP, Meher JG, Pandey N, Luqman S, Yadav KS, Chanda D. Enrichment, development, and assessment of Indian basil oil based antiseptic cream formulation utilizing hydrophiliclipophilic balance approach. Biomed Res Int. 2013;2013, Article ID 410686, 9 pages.

27. Sarkar S, Gupta S, Variyar PS, Sharma A, Singhal RS. Hydrophobic derivatives of guar gum hydrolyzate and gum arabic as matrices for microencapsulation of mint oil. Carbohydr Polym. 2013;95(1):177-82.

28. Mokhtari-Hosseini ZB, Vasheghani-Farahani E, HeidarzadehVazifekhoran A, Shojaosadati SA, Karimzadeh R, Khosravi Darani K. Statistical media optimization for growth and PHB production from methanol by a methylotrophic bacterium. Bioresour Technol. 2009;100(8):2436-43.

29. Muzzarelli RAA. Biomedical exploitation of chitin and chitosan via mechano-chemical disassembly, electrospinning, dissolution in imidazolium ionic liquids, and supercritical drying. Mar Drugs. 2011;9(9):1510-33.

30. Shojaee-Aliabadi S, Hosseini H, Mohammadifar MA, Mohammadi A, Ghasemlou M, Hosseini SM, et al. Characterization of kappa-carrageenan films incorporated plant essential oils with improved antimicrobial activity. Carbohydr Polym. 2014;101:582-91.

31. Teshome K, Gebre-Mariam T, Asres K, Perry F, Engidawork E. Toxicity studies on dermal application of plant extract of Plumbago zeylanica used in Ethiopian traditional medicine. J Ethnopharmacol. 2008;117(2):236-48. 